\title{
Efficacy and safety of nivolumab in Japanese patients with first recurrence of glioblastoma: an open-label, non-comparative study
}

\author{
Tomokazu Aoki $^{1}$ (D) Naoki Kagawa ${ }^{2} \cdot K$ Kazuhiko Sugiyama ${ }^{3} \cdot$ Toshihiko Wakabayashi $^{4} \cdot$ Yoshiki Arakawa $^{5}$. \\ Shigeru Yamaguchi ${ }^{6} \cdot$ Shota Tanaka $^{7} \cdot$ Eiichi Ishikawa $^{8} \cdot$ Yoshihiro Muragaki $^{9} \cdot$ Motoo Nagane $^{10}$. \\ Mitsutoshi Nakada ${ }^{11}$. Satoshi Suehiro ${ }^{12}$. Nobuhiro Hata ${ }^{13}$. Junichiro Kuroda ${ }^{14}$. Yoshitaka Narita ${ }^{15}$. \\ Yukihiko Sonoda ${ }^{16}$. Yasuo Iwadate ${ }^{17} \cdot$ Manabu Natsumeda ${ }^{18} \cdot$ Yoichi Nakazato $^{19} \cdot$ Hironobu Minami $^{20}$. \\ Yuki Hirata $^{21} \cdot$ Shunsuke Hagihara ${ }^{22} \cdot$ Ryo Nishikawa ${ }^{23}$
}

Received: 30 March 2021 / Accepted: 7 September 2021 / Published online: 29 September 2021

(c) The Author(s) 2021

\begin{abstract}
Background An open-label, non-comparative study assessed the efficacy and safety of nivolumab in Japanese patients with first recurrence glioblastoma.

Methods Patients with first recurrence of histologically confirmed World Health Organization Grade IV glioma, after treatment with temozolomide and radiotherapy, received nivolumab $3 \mathrm{mg} / \mathrm{kg}$ every 2 weeks until confirmed disease progression (Response Assessment in Neuro-Oncology criteria) or toxicity. Primary endpoint was 1-year overall survival rate assessed by Bayesian approach. The prespecified efficacy criterion was that the Bayesian posterior probability threshold for exceeding the 1-year overall survival of bevacizumab (34.5\%) from the Japanese phase 2 study (JO22506) would be 93\%.

Results Of the 50 enrolled patients, $44(88.0 \%)$ had recurrent malignant glioma (glioblastoma, gliosarcoma), and of these, $26(59.1 \%)$ had at least one measurable lesion at baseline. The Bayesian posterior mean 1-year overall survival (90\% Bayesian credible intervals) with nivolumab was 54.4\% (42.27-66.21), and the Bayesian posterior probability of exceeding the threshold of the 1-year overall survival rate of bevacizumab (34.5\%) was $99.7 \%$. Median (90\% confidence interval) overall and progression-free survival was 13.1 (10.4-17.7) and 1.5 (1.4-1.5) months, respectively. One partial response was observed (objective response rate $1 / 26$ evaluable patients [3.8\%]). Treatment-related adverse event rates were $14.0 \%$ for Grade $3-4$ and $2.0 \%$ for Grade 5; most adverse events resolved and were manageable.

Conclusions The 1-year overall survival with nivolumab monotherapy in Japanese patients with glioblastoma met the prespecified efficacy criterion. The safety profile of nivolumab was consistent with that observed in other tumor types.
\end{abstract}

Clinical Trial Registration JapicCTI-152967.

Keywords Bayesian approach $\cdot$ Bevacizumab $\cdot$ Clinical Trial $\cdot$ Phase II $\cdot$ Glioblastoma $\cdot$ Nivolumab $\cdot$ Programmed cell death

\section{Introduction}

In Japan, approximately 4000-5000 new cases of gliomas are reported each year [1,2]. Despite treatment, patient outcomes remain poor in Japan [3], with a 5-year overall survival (OS) rate of $15.5 \%$, a median OS of 18 months, and a local recurrence rate of $51 \%$ recorded [4]. Different treatment guidelines have been created for recurrent glioblastoma across the

Tomokazu Aoki

totorolangdom@yahoo.co.jp

Extended author information available on the last page of the article globe, but no standard treatment regimen has been established [5-7]. Currently, surgical re-excision or systemic and local chemotherapy with temozolomide, nitrosoureas, bevacizumab, or stereotactic irradiation to control localized lesions may be considered [7]. Bevacizumab was approved in Japan for recurrent glioblastoma based on a phase 2 trial (JO22506) of bevacizumab monotherapy in which the 6-month progression-free survival (PFS) rate was $33.9 \%$, the 1-year OS rate was $34.5 \%$, and the median OS was 10.5 months in Japanese patients with recurrent glioblastoma [8]. Although bevacizumab is often used for the treatment of recurrent glioblastoma, its clinical benefit is transient and variable $[9,10]$. 
Recent advances in immuno-oncology provide evidence for the efficacy of programmed cell death-1 (PD-1)/programmed death-ligand 1 (PD-L1) blockade for a subset of cancers [11-15]. Several preclinical studies have demonstrated PD-L1 expression status in human glioma tissues $[11,16]$; however, evidence of the efficacy of PD-1/PD-L1 antibody immunotherapy in glioblastoma is limited. Nivolumab, an anti-PD-1 monoclonal antibody [17], is approved in the United States, European Union, and Asia for the treatment of several cancer types [18]. Considering the link between PD-L1 expression and glioblastoma, nivolumab was hypothesized to be a potential therapeutic agent for the treatment of glioblastoma. Preliminary studies and case reports have shown benefits with nivolumab for glioblastoma; however, further research is needed [11]. The CheckMate 143 study (NCT02017717) evaluated the efficacy and safety of nivolumab vs bevacizumab in non-Japanese patients with recurrent glioblastoma [19]. Although the CheckMate 143 study (369 patients) did not demonstrate improved OS with nivolumab compared with bevacizumab (median OS, 9.8 vs 10 months), the median duration of response in evaluable patients was longer in the nivolumab group (11.1 months) than the bevacizumab group (5.3 months) [19]. These data suggest that nivolumab may offer some benefit to patients with recurrent glioblastoma. The objective of the current study (ONO-4538-19), run in parallel with the non-Japanese CheckMate 143 study, was to evaluate the efficacy and safety of nivolumab in Japanese patients with recurrent glioblastoma.

\section{Materials and methods}

\section{Study design}

This multicenter, open-label, non-comparative, non-randomized, phase 2 study evaluated the efficacy and safety of nivolumab in Japanese patients. The protocol was approved by the institutional review board of each study site $(n=20)$, was conducted between October 2015 and April 2019, and consisted of screening, treatment, and follow-up periods. The cut-off date for the data in this report is December 2017.

The study was conducted in accordance with the principles of the Declaration of Helsinki and Good Clinical Practice guidelines, as well as all local laws and regulations. The study was registered at the Japan Pharmaceutical Information Center (www.japic.org; JapicCTI-152967). All patients provided written informed consent.

\section{Patients}

Adults (aged $\geq 20$ years) with a first recurrence of histologically confirmed World Health Organization Grade IV malignant glioma (glioblastoma or gliosarcoma) confirmed by magnetic resonance imaging per Response Assessment in Neuro-Oncology (RANO) criteria or by histopathological evidence were enrolled. Patients had received first-line treatment with temozolomide plus radiotherapy (standard focally directed only), had a Karnofsky Performance Status (KPS) $\geq 70$, and a life expectancy of $\geq 12$ weeks. Patients with no measurable lesion or those with an interval of $\geq 28$ days post-surgical resection after the first recurrence were also eligible.

Main exclusion criteria included patients with secondary glioblastoma (i.e., progress from low-grade diffuse astrocytoma, anaplastic astrocytoma, etc.) or extracranial metastatic or leptomeningeal disease, patients with multiple primary cancers, patients receiving treatments other than surgical therapy for recurrent glioblastoma, and patients with escalating or chronic supraphysiological doses of corticosteroids for disease control. Also excluded were patients receiving prior treatment with carmustine wafers (except when administered as first-line treatment and $\geq 180$ days prior to randomization), bevacizumab, other monoclonal antibodies targeting vascular endothelial growth factor receptors, or antiangiogenic therapy, and patients with prior PD-1/ PD-L1 or cytotoxic T-lymphocyte-associated protein targeted therapies.

\section{Treatment}

Nivolumab $3 \mathrm{mg} / \mathrm{kg}$ was administered intravenously for approximately $60 \mathrm{~min}$ on day 1 of each 2-week cycle (same regimen as CheckMate 143) [19], with the first dose administered within 7 days of enrollment and at least a 10-day interval between doses. Treatment was continued until confirmed disease progression (by RANO criteria) or development of toxicity. However, continued treatment was permitted until re-confirmation of progression approximately 3 months after initially meeting RANO progressive disease (PD) criteria. There were no restrictions on using bevacizumab to treat worsening glioblastoma-associated symptoms post-second recurrence or disease progression confirmation.

\section{Endpoints and assessments}

The primary efficacy endpoint was the 1-year survival rate, defined as the proportion of patients alive at 1 year since day 1 of treatment administration. Other endpoints included: best overall response per RANO criteria-the percentage of patients with complete response (CR) sustained for at least 4 weeks, partial response (PR) sustained for at least 4 weeks, stable disease (SD), PD, and non-evaluable disease; objective response rate (ORR), defined as the percentage of patients whose best overall response was a confirmed CR or PR (central and investigator assessment); OS; PFS 
Table 1 Baseline and clinical characteristics of the total study population
Nivolumab $(N=50)$

Sex

Male

34 (68.0)

Female

$16(32.0)$

Age, years

$<65$

$37(74.0)$

$65-<75$

$12(24.0)$

$\geq 75$

$1(2.0)$

Karnofsky performance status

$100 \%$

$90 \%$

$18(36.0)$

$80 \%$

$11(22.0)$

$70 \%$

$16(32.0)$

MGMT gene promoter methylation

Unmethylated

10 (20.0)

Methylated

$12(24.0)$

Unknown

2 (4.0)

Not performed

$26(52.0)$

Histopathological diagnosis (central review)

Glioblastoma

$43(86.0)$

Gliosarcoma

$1(2.0)$

$6(12.0)$

Others $^{\mathrm{a}}$

$9.2(2.0-61.9)$

Corticosteroid use at baseline ${ }^{b}$

No

Yes

$<4 \mathrm{mg} /$ day

$6(12.0)$

$\geq 4 \mathrm{mg} /$ day

0

Prior systemic therapy

No

0

Yes

$50(100.0)$

Temozolomide

$50(100.0)$

Carmustine wafers

$12(24.0)$

Others

$2(0-4)$

Patients with $\geq 1$ measurable lesion

No

$13(26.0)$

Yes

$37(74.0)$

Sum of products of maximum perpendicular diameters of measurable lesions ${ }^{\mathrm{c}}$ (investigator review), median (range)

$978.6(110.0-3215.9)$

PD-L1 status

$1 \%$ positive

$18(36.0)$

$1 \%$ negative

Not measured

Values are $n(\%)$, unless otherwise stated

${ }^{a}$ Other histopathological diagnoses (based on central review assesment) included: anaplastic oligoastrocytoma $(n=1)$, anaplastic astrocytoma $(n=1)$, a diagnosis compatible with anaplastic astrocytoma $(n=2)$, no evidence of tumor $(n=1)$, and slight infiltration of isocytrate dehydrogenase 1-mutated glioma cells $(n=1)$

${ }^{\mathrm{b}}$ Based on average corticosteroid use 5 days prior to start of dosing in dexamethasone equivalents

${ }^{\mathrm{c}}$ Analyzed only patients with $\geq 1$ measurable lesion

$M G M T \mathrm{O}^{-6}$ methylguanine-DNA methyltransferase, $P D-L 1$ programmed death-ligand 1 
(central and investigator assessment); and percentage change and maximum percentage change from baseline in sum of the products of maximal perpendicular diameters (SPD) of measurable lesions (investigator assessment).

Subgroup analyses of OS based on age, sex, KPS, $O^{6}$-methylguanine-DNA methyltransferase (MGMT) methylation status, PD-L1 status, and corticosteroid use were conducted. Safety endpoints included the type, frequency, severity, and seriousness of adverse events (AEs) and the causal relationship with nivolumab. Types of AEs were assessed according to the Medical Dictionary for Regulatory Activities, version 20.1; AE grades were classified according to the Japanese translation of the National Cancer Institute Common Terminology Criteria for Adverse Events, version 4.0.

\section{Statistical methods}

Efficacy was assessed using the full analysis set (FAS), which consisted of patients who received at least one dose of nivolumab, were compliant with Good Clinical Practice, and had Grade IV malignant glioma confirmed by central pathological review. Safety endpoints were assessed using the safety analysis set (SAS), which consisted of patients who received at least one dose of nivolumab.

A Bayesian approach [20-22] was used to assess the primary endpoint. At the time this study was planned, CheckMate 143 results were not available; therefore, the efficacy criterion of this study was based on the Japanese JO22506 study [8]. The prespecified efficacy criterion was that the posterior probability of the 1-year survival rate with nivolumab in this study exceeding the threshold 1 -year survival rate with bevacizumab (34.5\% [90\% confidence interval (CI) 20.0-49.0]) from the JO22506 study would be more than $93 \%$. Details of the probability density function are provided in Supplementary Material (Online Resource 1). To compute a $90 \%$ Bayesian credible interval, the quantiles of the posterior distribution used in this study were 0.05 and 0.95 . Assuming the 1 -year survival rate of nivolumab would be $49.0 \%$ based on the 1-year survival rate in the JO22506 study, and using the same assumed hazard ratio (HR) as the CheckMate 143 study (0.67) [19], the estimated sample size was 42 patients calculated using the Bayesian method [23]. However, to allow for sufficient patient numbers with confirmation of glioma diagnosis by central pathological review, a target sample size of 45 was selected. A uniform prior distribution of Beta $(1,1)$ was selected for the prior distribution of the 1-year OS rate.

Best overall response and the ORR and their 90\% CIs were calculated using the Clopper-Pearson method. Median OS and PFS with its 90\% CIs, as well as OS and PFS rates at months $6,12,18$, and 24 , were calculated using the Kaplan-Meier method. The CIs of medians were calculated using the Brookmeyer-Crowley method [24], and the CIs of rates were derived based on Greenwood's formula using double logarithmic transformation. HRs and $90 \%$ CIs were estimated using the Cox unstratified proportional hazards model. Percentage change and the maximum percentage change in the SPD of measurable lesions were plotted for each patient using spider plots and waterfall plots, respectively. No additional analyses were conducted for missing data, nor were adjustment analyses by covariates performed.

\section{Results}

\section{Baseline characteristics}

Of 50 patients enrolled (Table 1), 44 patients (88.0\%) had recurrent malignant glioma (glioblastoma and gliosarcoma). A total of $37(74.0 \%)$ patients had at least one measurable lesion at baseline, and corticosteroids were used by six (12.0\%) patients at a dose $<4 \mathrm{mg} /$ day. A total of 46 patients

Table 2 Primary endpoint: 1-year survival rate (FAS)

\begin{tabular}{|c|c|}
\hline & Nivolumab $(n=44)^{\mathrm{a}}$ \\
\hline Posterior mean of the 1-year survival rate, $\%$ & 54.4 \\
\hline Posterior mode & 54.5 \\
\hline Posterior variance & 0.53 \\
\hline $90 \%$ Bayesian credible intervals & $42.27-66.21$ \\
\hline $\begin{array}{l}\text { Posterior probability that the result of the study } \\
\text { exceeds the threshold 1-year survival rate, } \%\end{array}$ & 99.7 \\
\hline \multicolumn{2}{|l|}{${ }^{\mathrm{a}}$ Includes one patient who had gliosarcoma } \\
\hline \multicolumn{2}{|c|}{$\begin{array}{l}{ }^{\mathrm{b}} \text { The } 1 \text {-year survival rate of } 34.5 \% \text { in the JO22506 study (phase } 2 \\
\text { study of single-agent bevacizumab in Japanese patients with recurrent } \\
\text { glioblastoma) was selected as the threshold 1-year survival rate }\end{array}$} \\
\hline \multicolumn{2}{|c|}{ FAS full analysis set } \\
\hline
\end{tabular}

Table 3 Best overall response per RANO criteria (FAS)

\begin{tabular}{lll}
\hline Best overall response & \multicolumn{2}{l}{ Nivolumab $(n=44)$} \\
\cline { 2 - 3 } & Central review & Investigator review \\
\hline $\mathrm{CR}$ & 0 & 0 \\
$\mathrm{PR}$ & $1(2.3)$ & $2(4.5)$ \\
$\mathrm{SD}$ & $2(4.5)$ & $5(11.4)$ \\
$\mathrm{PD}$ & $23(52.3)$ & $24(54.5)$ \\
$\mathrm{NE}$ & $18(40.9)$ & $13(29.5)$ \\
No measurable lesion & $16(36.4)$ & $11(25.0)$ \\
Other reasons & $2(4.5)^{\mathrm{a}}$ & $2(4.5)$ \\
\hline
\end{tabular}

Values are $n(\%)$

${ }^{a}$ Includes two patients who did not have a central radiologic review $C R$ complete response, FAS full analysis set, $N E$ not estimable, $P D$ progressive disease, $P R$ partial response, RANO Radiologic Assessment in Neuro-Oncology criteria, $S D$ stable disease 
(92\%) discontinued treatment owing to disease progression (78\%), dosing delay lasting $>6$ weeks $(4 \%)$, investigator decision $(4 \%)$, or other reasons $(6 \%)$.

For patients included in the FAS $(N=44), 26(59.1 \%)$ patients had at least one measurable lesion at baseline determined by central pathological review, and corticosteroid use was low with only four patients $(9.1 \%)$ using corticosteroids at a dose $<4 \mathrm{mg} /$ day.

\section{Efficacy}

In the FAS, the posterior mean (90\% Bayesian credible intervals) of the 1-year survival rate with nivolumab monotherapy was $54.4 \%$ (42.27-66.21), and the posterior probability of exceeding the prespecified threshold 1-year survival rate (i.e., 34.5\%) was $99.7 \%$ (Table 2). Thus, the 1-year survival rate with nivolumab met the efficacy criterion prespecified for this study, and the primary endpoint was met.

Best overall response with central review was PR (one patient; $2.3 \%$ ) resulting in an ORR of $1 / 26(3.8 \%)$ in patients with measurable lesions (Table 3). Median duration of response for the one patient with $\mathrm{PR}$ was 5.5 months, with a time to response of 2.8 months. Best overall response with investigator review was PR (two patients; $4.5 \%$ ). SD was observed for $4.5 \%$ and $11.4 \%$ of patients with central and investigator reviews, respectively; no patient had a CR. There was good agreement between central and investigator
Fig. 1 a Overall survival and b progression-free survival by central assessment. Vertical dashes represent censored observations. $C I$ confidence interval, $m O S$ modified overall survival, $m P F S$ modified progression-free survival, $P F S$ progression-free survival

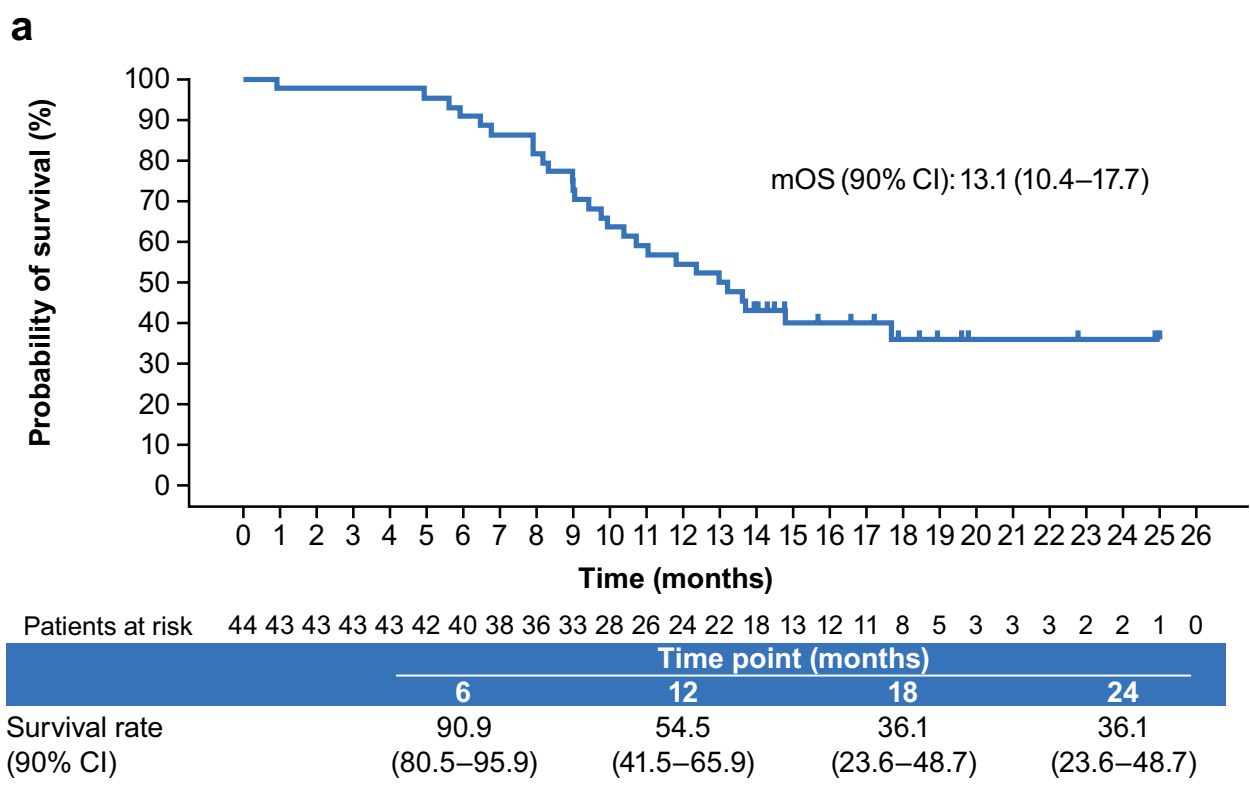

b

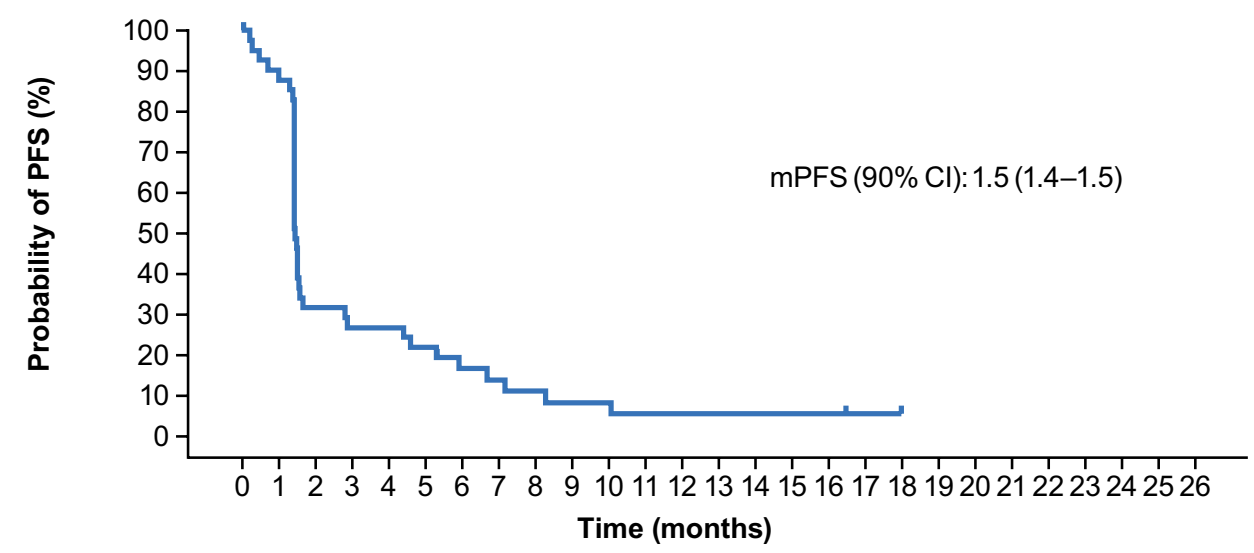

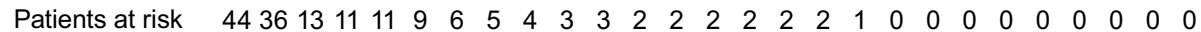

\begin{tabular}{lcccc} 
& \multicolumn{4}{c}{ Time point (months) } \\
\cline { 2 - 5 } & 6 & 12 & 18 & 24 \\
PFS & 16.7 & 5.6 & - & - \\
$(90 \% \mathrm{Cl})$ & $(8.4-27.4)$ & $(1.4-14.0)$ & $(-,-)$ & $(-,-)$
\end{tabular}


reviews for the proportion of patients with PD (52.3\% and $54.5 \%$, respectively).

Median (90\% CI) OS was 13.1 months (10.4-17.7) (Fig. 1a), and OS rates at 6, 12, 18, and 24 months were $90.9 \%, 54.5 \%, 36.1 \%$, and $36.1 \%$, respectively. Median (90\% CI) PFS by central assessment was 1.5 months (1.4-1.5) (Fig. 1b). The size of the measurable lesion decreased in approximately $30 \%$ of patients with measurable lesions, and the antitumor effects were sustained in some of the patients with reduced measurable lesions (Fig. 2). Following nivolumab treatment, the switching rate to bevacizumab for the treatment of secondary recurrence was $65.9 \%$ (29/44 patients).

Subgroup analysis of OS was consistent with the primary analysis of OS (Table 4). Items that showed a measurable difference in median OS of more than 1 month between subgroups were KPS, MGMT promoter methylation, and PD-L1 status. There was a trend for longer median (90\% CI) OS with increasing KPS score (KPS $100 \%$ or $90 \%$ vs $80 \%$ or 70\%: HR, 0.55 ; 90\% CI $0.29-1.05$ ) and in patients with evidence of $M G M T$ methylation (methylation vs unmethylation: HR $0.44 ; 90 \%$ CI 0.17-1.15), whereas, it was shorter for patients with PD-L1 positivity (1\% cut-off) (PD-L1 positive vs PD-L1 negative: HR 3.03; 90\% CI 1.44-6.36). For subgroups of female sex, age $\geq 65$ years, and patients with baseline corticosteroid use, the median OS was not reached. In the majority of patients, specimens obtained at operation for newly diagnosed glioblastoma were used for PD-L1 immunohistochemistry analyses.

\section{Safety}

In the SAS $(N=50), 90 \%$ of patients treated with nivolumab experienced AEs, with treatment-related adverse events (TRAEs) and serious adverse events related to nivolumab observed in $48.0 \%$ (Grades 3-4, 14.0\%) and 12.0\% (Grades $3-4,10 \%$ ) of patients, respectively (Table 5 ). The most common AEs (frequency $\geq 10 \%$ ) were fever, headache, lymphocytopenia, constipation, nasopharyngitis, increased $\gamma$-glutamyl transferase, insomnia, and brain edema.
Fig. 2 a Percentage change and b maximum percentage change from baseline in SPD of measurable lesions by investigator assessment. Panel a includes measurements from baseline to follow-up (including measurements after PD documentation). $N=31$ patients; 13 patients who had a best overall response of $\mathrm{NE}^{\mathrm{a}}$ were excluded. Panel $\mathbf{b}$ includes measurements from baseline up to PD documentation. $N=29 ; 13$ patients who had a best overall response of $\mathrm{NE}^{\mathrm{a}}$ and two patients with no MRI prior to a diagnosis of PD based on clinical deterioration were excluded. ${ }^{\text {a}}$ Where NE was owing to no measurable lesion available by investigator review or no evaluable MRI scans available after dosing. $M R I$ magnetic resonance imaging, $N E$ not estimable, $P D$ progressive disease, $S P D$ sum of the products of maximal perpendicular diameter a

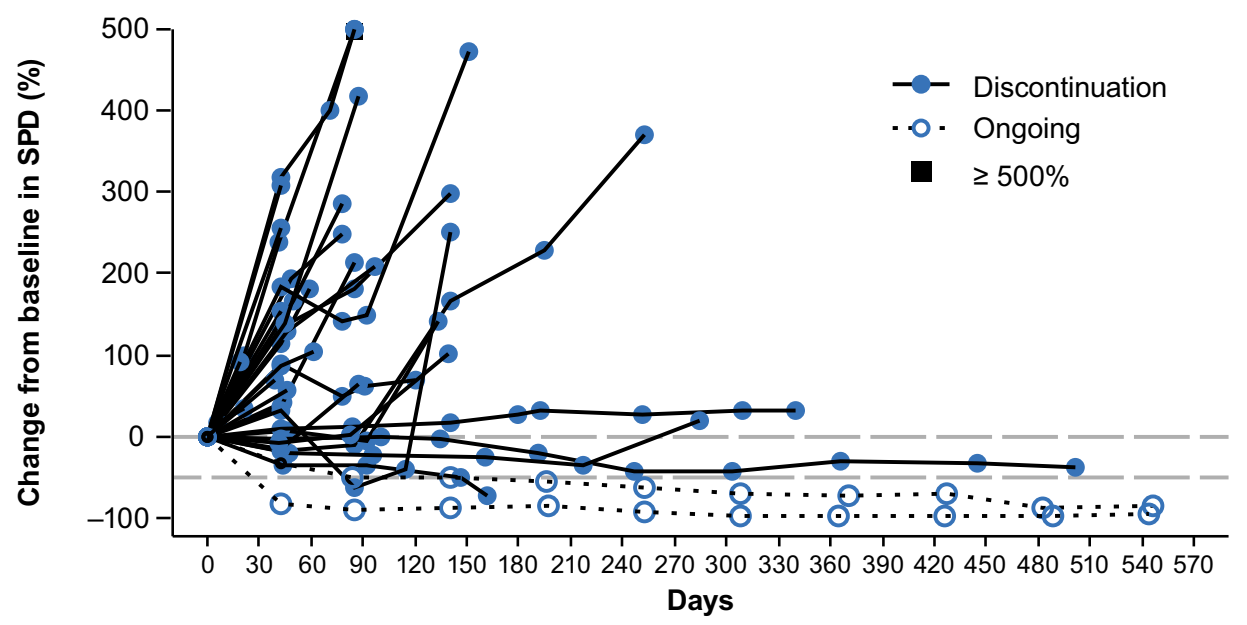

b

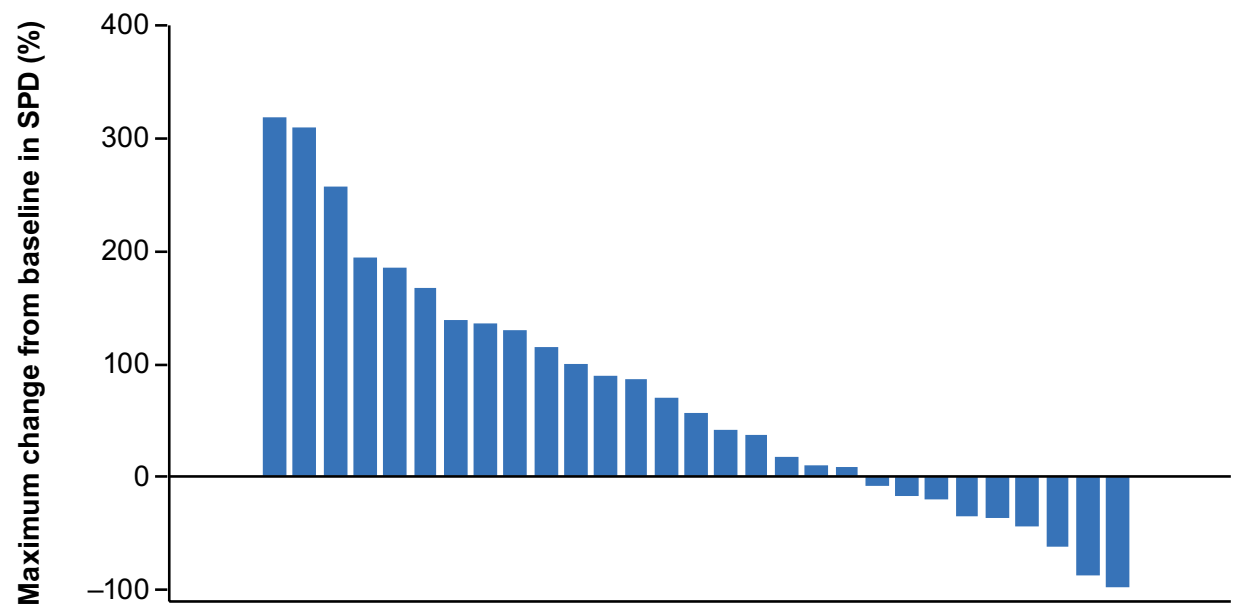


Table 4 Subgroup analyses for OS (FAS)

\begin{tabular}{lll}
\hline Subgroup & \multicolumn{2}{l}{ Nivolumab $(n=44)$} \\
\cline { 2 - 3 } & $n$ & mOS $(90 \%$ CI $)$, months \\
\hline Sex & & \\
Male & 31 & $12.4(9.8-13.7)$ \\
Female & 13 & $-(8.3,-)$ \\
Age, years & & \\
$<65$ & 32 & $11.4(9.0-13.6)$ \\
$\geq 65$ & 12 & $-(9.4,-)$ \\
Karnofsky Performance Status & & \\
$100 \%$ & 5 & $17.7(8.2,-)$ \\
$90 \%$ & 16 & $14.0(9.8,-)$ \\
$80 \%$ & 11 & $12.4(7.9-13.7)$ \\
$70 \%$ & 12 & $10.5(6.8,-)$ \\
$M G M T$ gene promoter methylation & & \\
Unmethylated & 7 & $9.0(5.6-12.4)$ \\
Methylated & 11 & $14.8(9.0,-)$ \\
Unknown & 2 & $10.0(6.8-13.6)$ \\
Not performed & 24 & $15.7(10.7,-)$ \\
Corticosteroid use at baseline & & \\
No & 40 & $13.1(9.9-17.7)$ \\
Yes & 4 & $-(5.0,-)$ \\
1\% PD-L1 status & & \\
Positive & 17 & $10.7(9.0-13.0)$ \\
Negative & 18 & $17.7(13.6,-)$ \\
Not measured & 9 & $8.2(6.5,-)$ \\
\hline & &
\end{tabular}

- indicates endpoint "not reached"

$C I$ confidence interval, FAS full analysis set, $M G M T \mathrm{O}^{-6}$ methylguanine-DNA methyltransferase, $m O S$ median overall survival, $O S$ overall survival, $P D-L 1$ programmed death-ligand 1

Drug-related AEs leading to treatment discontinuation were observed in four patients $(8.0 \%)$. The total Grade 3-5 TRAE rate was $16.0 \%$. Most AEs resolved and were manageable. Grade 5 rhabdomyolysis was observed in one patient $(2.0 \%)$ during cycle 2 . The patient contracted influenza 9 days after receiving study drug, with persistent pyrexia, followed by the occurrence of acute kidney injury due to rhabdomyolysis; the patient developed a respiratory disorder owing to pulmonary congestion and died.

\section{Discussion}

The results of this prospective study indicate that nivolumab may have clinical activity with evidence of acceptable toxicity in Japanese patients with recurrent glioblastoma. The safety profiles of nivolumab in this study were consistent with that of previous studies of nivolumab in multiple tumor types [25-30]. No new safety signals were identified. Implementing the Bayesian approach, the results of this study suggest that nivolumab may be at least as effective as bevacizumab in the treatment of recurrent glioblastoma in Japanese patients. However, a direct comparison of the efficacy of nivolumab in this study with that of bevacizumab in the JO22506 study is difficult considering the different patient background factors of each study (discussed in detail below), and the fact that in this study more than $60 \%$ of patients switched to bevacizumab treatment upon disease progression.

In this study, the posterior mean $(90 \%$ Bayesian credible intervals) 1-year survival rate with nivolumab was $54.4 \%$ (42.27-66.21), and the observed posterior probability of exceeding the 1-year survival rate of $34.5 \%$ for bevacizumab in the JO22506 study [8] was estimated to be $99.7 \%$, exceeding the prespecified threshold of $93 \%$. These results may support the probability that nivolumab might be more effective than bevacizumab in a Japanese population. It is important to note that the threshold 1-year survival rate (34.5\%) selected for this study was based on the JO22506 study, which included patients with both first and second recurrence glioblastoma [8], whereas, only patients with first recurrence were included in this study. After the planning of the current study was completed, the 1-year survival rate with bevacizumab in the CheckMate 143 study became available, which was $42.0 \%$ [19].

In this phase 2 study, both the 1-year survival rate $(54.5 \%)$ and median OS (13.1 months) were numerically higher than that observed with bevacizumab (42.0\% and 10.0 months, respectively) and nivolumab (41.8\% and 9.8 months, respectively) in the CheckMate 143 study [19]. In CheckMate 143, the response rate was lower $(7.8 \%$ vs $23.1 \%)$, and median PFS was shorter (1.5 vs 3.5 months, respectively) with nivolumab compared with bevacizumab, and there was no survival benefit (HR 1.04; 95\% CI 0.83-1.30). The observed differences between these two studies may be attributed to the multiple nivolumab injections or the switch to thirdline therapies (65.9\% switched to bevacizumab) from early nivolumab discontinuations in the current study, or to differences in baseline characteristics, such as corticosteroid use or MGMT methylation. The proportion of patients with corticosteroid use at baseline in this study was low $(9.1 \%)$ compared with the proportion of patients in the nivolumab group in the CheckMate 143 study (39.7\%). However, for patients with no corticosteroid use at baseline, the median OS with nivolumab was comparable in this study and in CheckMate 143 (13.1 vs 12.6 months, respectively), which was slightly higher than with bevacizumab in CheckMate 143 (11.8 months) [19].

Bevacizumab has established efficacy in treating recurrent glioblastomas [8, 31, 32]. In this study, patients responded well to nivolumab and achieved longer OS (13.1 months) than that reported for bevacizumab (10.5 months) in Japanese patients with recurrent glioblastoma (JO22506) [8]; 
Table 5 Summary of adverse events (SAS)

\begin{tabular}{lll}
\hline Adverse events, $n(\%)$ & All grades $(n=50)$ & Grades 3-4 $(n=50)$ \\
\hline All causality & & \\
All AEs & $45(90.0)$ & $24(48.0)$ \\
SAE & $16(32.0)$ & $11(22.0)$ \\
AEs leading to discontinuation & $7(14.0)$ & $4(8.0)$ \\
AEs occurring in $\geq 10 \%$ of patients & & \\
Pyrexia & $10(20.0)$ & 0 \\
Headache & $9(18.0)$ & 0 \\
Lymphocyte count decreased & $9(18.0)$ & $5(10.0)$ \\
Constipation & $8(16.0)$ & 0 \\
Nasopharyngitis & $7(14.0)$ & 0 \\
$\gamma$-Glutamyl transferase increased & $5(10.0)$ & 0 \\
Insomnia & $5(10.0)$ & 0 \\
Brain edema & $5(10.0)$ & $1(2.0)$ \\
TRAEs & & $7(14.0)$ \\
All TRAEs & $24(48.0)$ & $5(10.0)$ \\
Serious TRAEs & $6(12.0)$ & $2(4.0)$ \\
TRAEs leading to discontinuation & $4(8.0)$ & 0 \\
TRAEs occurring in $\geq 2$ patients & & 0 \\
$\gamma$-Glutamyl transferase increased & $4(8.0)$ & $1(2.0)$ \\
Lymphocyte count decreased & $3(6.0)$ & $1(2.0)$ \\
Brain edema & $2(4.0)$ & 0 \\
Diarrhea & $2(4.0)$ & 0 \\
Pyrexia & $2(4.0)$ & $1(2.0)$ \\
Hypopituitarism & $2(4.0)$ & \\
Rash maculo-papular & $2(4.0)$ & \\
\hline
\end{tabular}

$A E$ adverse event, $S A E$ serious adverse event, $S A S$ safety analysis set, TRAE treatment-related adverse event however, a total of $65.9 \%$ of patients in this study switched to bevacizumab upon disease progression (median PFS 1.5 months), and this may have impacted the 1-year OS rate. PFS and ORR were not improved with nivolumab in this study compared with bevacizumab (Online Resource 2). In addition, the lower use of corticosteroids and higher proportion of younger patients at baseline in the current study, and inclusion of patients with a second recurrence in the JO22506 study, may have influenced the difference in survival results. Median OS with bevacizumab treatment has been shown to be slightly influenced by corticosteroid use [19]. Patients in the JO22506 study with a second recurrence likely required corticosteroids to maintain a KPS $\geq 70 \%$; thus, they may have had a worse prognosis than the patients included in the current study.

The use of a Bayesian approach allowed appraisal of the results of parallel trials in glioblastoma, which may assist in future clinical decision-making. Using prespecified, datadriven, and scientifically based success criteria resulted in a well-designed study. In addition, the inclusion of subgroup analyses permitted the assessment of clinical and tumor characteristics to predict the clinical activity of nivolumab. This study showed consistency with CheckMate 143 with regard to potential benefits in selected subgroups, such as patients with no baseline corticosteroid use and those with MGMT methylation, in whom the median OS was longer with nivolumab than with bevacizumab. However, the success criterion was based on only one endpoint, and the effects of other endpoints were not considered. Moreover, the PFS with nivolumab was shown to be less than 2 months. Other limitations of the study included the lack of a direct comparator, the small sample size, and the lack of generalizability of the results to non-Japanese patients. Therefore, the results of this study should be verified in larger comparative trials.

In conclusion, this study demonstrated that nivolumab has acceptable toxicity with potential clinical activity, according to preset criteria, in Japanese patients with glioblastoma with first recurrence. However, PFS and ORR were not improved compared with the JO22506 study.

Supplementary Information The online version contains supplementary material available at https://doi.org/10.1007/s10147-021-02028-1.

Acknowledgements The authors would like to thank all study participants. The authors would also like to thank all the staff who assisted with various aspects during the clinical trial. Medical writing support was provided by Sandra Kurian, MPharm, and Tania Dickson, PhD, 
CMPP, of ProScribe-Envision Pharma Group, and was funded by Ono Pharmaceutical Co., Ltd. and Bristol-Myers Squibb.

Author contributors All authors participated in the drafting, critical revision, and approval of the final version of the manuscript. Tomokazu Aoki, Hironobu Minami, Yuki Hirata, Shunsuke Hagihara, and Ryo Nishikawa were involved in the study design. Hironobu Minami, Yuki Hirata, and Shunsuke Hagihara interpreted the study results, and Y. Nakazato contributed to pathology review. All authors except Yoichi Nakazato, Hironobu Minami, Yuki Hirata, and Shunsuke Hagihara were investigators in the study and involved in data collection. Shunsuke Hagihara conducted the statistical analysis.

Funding This work was supported by Ono Pharmaceutical Co., Ltd. and Bristol-Myers Squibb. The study sponsor was involved in the study design, writing of the report, and in the decision to submit the article for publication.

\section{Declarations}

Conflict of interest T. Aoki, N. Kagawa, T. Wakabayashi, S. Yamaguchi, E. Ishikawa, S. Suehiro, J. Kuroda, Y. Iwadate, M. Natsumeda, Y. Nakazato, and S. Hagihara have no conflicts of interest to declare. K. Sugiyama has received research funding from Ono Pharmaceutical Co., Ltd., Daiichi-Sankyo Co., Ltd., and Sumitomo Dainippon Pharma Co. Ltd. Y. Arakawa has received research funding from Ono Pharmaceutical Co., Ltd., Siemens, Philips, Chugai Pharmaceutical Co., Ltd., Daiichi-Sankyo Co., Ltd., and Taiho Pharmaceutical Co., Ltd.; and honoraria from Chugai Pharmaceutical Co., Ltd., Eisai Co., Ltd., and Daiichi-Sankyo Co., Ltd. S. Tanaka has received research funding from Eisai Co., Ltd., Ono Pharmaceutical Co., Ltd., and Sumitomo Dainippon Pharma Co. Ltd. Y. Muragaki has received lecture fees, honoraria, or other fees from Eisai Co., Ltd. and Novocure Limited. M. Nagane has received lecture fees, honoraria, or other fees from MSD and Ono Pharmaceutical Co., Ltd.; has received research funding from Toray Industries, Inc., AbbVie Inc., and Ono Pharmaceutical Co., Ltd.; and has received scholarship (incentive) endowments or research grants from Eisai Co., Ltd., Pfizer, Nippon Kayaku Co., Ltd., and Chugai Pharmaceutical Co., Ltd. M. Nakada has received research funding from Ono Pharmaceutical Co., Ltd.; and has received scholarship (incentive) endowments or research grants from Otsuka Pharmaceutical Co., Ltd., Stryker Corporation, Eisai Co., Ltd., MSD, and Chugai Pharmaceutical Co., Ltd. N. Hata has received research funding from Daiichi-Sankyo Co., Ltd. Y. Narita has received research funding from Eisai Co., Ltd., Ono Pharmaceutical Co., Ltd., AbbVie Inc., Bayer AG, Sumitomo Dainippon Pharma Co. Ltd., Taiho Pharmaceutical Co., Ltd., and Daiichi-Sankyo Co., Ltd. Y. Sonoda has received scholarship (incentive) endowments or research grants from Eisai Co., Ltd., Otsuka Pharmaceutical Co., Ltd., and Fujifilm. H. Minami has received lecture fees, honoraria, or other fees from Daiichi-Sankyo Co., Ltd. and Ono Pharmaceutical Co., Ltd.; has received research funding from Amgen Astellas BioPharma K.K., Bayer AG, Bristol-Myers Squibb, Daiichi-Sankyo Co., Ltd., Novartis, Pfizer, and Ono Pharmaceutical Co., Ltd.; and has received scholarship (incentive) endowments or research grants from Astellas, Bayer AG, Bristol-Myers Squibb, Chugai Pharmaceutical Co., Ltd., Daiichi-Sankyo Co., Ltd., Kyowa Kirin, Eli Lilly, Ono Pharmaceutical Co., Ltd., Pfizer, Taiho Pharmaceutical Co., Ltd., and Takeda Pharmaceutical Company Limited. Y. Hirata is an employee and shareholder at Ono Pharmaceutical Co., Ltd. R. Nishikawa has received manuscript fees from Eisai Co., Ltd.; has received research funding from AbbVie Inc., Daiichi-Sankyo Co., Ltd., Ono Pharmaceutical Co., Ltd., Toray Industries, Inc., and Medi-Physics, Inc.; and has received scholarship (incentive) endowments or research grants from Chugai Pharmaceutical Co., Ltd. and MSD.
Open Access This article is licensed under a Creative Commons Attribution 4.0 International License, which permits use, sharing, adaptation, distribution and reproduction in any medium or format, as long as you give appropriate credit to the original author(s) and the source, provide a link to the Creative Commons licence, and indicate if changes were made. The images or other third party material in this article are included in the article's Creative Commons licence, unless indicated otherwise in a credit line to the material. If material is not included in the article's Creative Commons licence and your intended use is not permitted by statutory regulation or exceeds the permitted use, you will need to obtain permission directly from the copyright holder. To view a copy of this licence, visit http://creativecommons.org/licenses/by/4.0/.

\section{References}

1. Narita Y (2012) Epidemiology and standard therapy in gliomas. Jpn J Neurosurg 21(3):184-191. https://doi.org/10.7887/jens.21. 184

2. Narita Y, Shibui S, Committee of Brain Tumor Registry of Japan supported by the Japan Neurosurgical Society (2015) Trends and outcomes in the treatment of gliomas based on data during 2001-2004 from the Brain Tumor Registry of Japan. Neurol Med Chir (Tokyo) 55(4):286-295. https://doi.org/10. 2176/nmc.ra.2014-0348

3. Osuka S, Van Meir EG (2017) Overcoming therapeutic resistance in glioblastoma: the way forward. J Clin Invest 127(2):415-426. https://doi.org/10.1172/JCI89587

4. Brain Tumor Registry of Japan (2005-2008) (2017). Neurol Med Chir (Tokyo) 57(Suppl 1):9-102. https://doi.org/10.2176/ nmc.sup.2017-0001

5. Stupp R, Brada M, van den Bent MJ et al (2014) High-grade glioma: ESMO clinical practice guidelines for diagnosis, treatment and follow-up. Ann Oncol 25(suppl_3):93-101. https:// doi.org/10.1093/annonc/mdu050

6. Nishikawa R (2010) Standard therapy for glioblastoma-a review of where we are. Neurol Med Chir 50(9):713-719. https://doi.org/ $10.2176 /$ nmc. 50.713

7. Japanese Society of Cancer Therapy, Japanese Society of Neurosurgery, Japanese Society of Brain Oncology (2016) Adult glioblastoma (GBM) guidelines - CQ6 how to treat adult recurrent glioblastoma? The Japan Society for Neuro-Oncology. https:// www.jsn-o.com/guideline3/CQ/006.html. Accessed Apr 24, 2020

8. Nagane M, Nishikawa R, Narita Y et al (2012) Phase II study of single-agent bevacizumab in Japanese patients with recurrent malignant glioma. Jpn J Clin Oncol 42(10):887-895. https://doi. org/10.1093/jjco/hys121

9. Li Y, Ali S, Clarke J, et al (2017) Bevacizumab in recurrent glioma: patterns of treatment failure and implications. Brain Tumor Res Treat 5(1):1-9. https://doi.org/10.14791/btrt.2017.5.1.1

10. Martinez NL, Glass J, Shi W (2019) Bevacizumab in recurrent glioblastoma. Transl Cancer Res 8(Suppl 2):S162-S163. https:// doi.org/10.21037/tcr.2018.12.33

11. Chen RQ, Liu F, Qiu XY et al (2019) The prognostic and therapeutic value of PD-L1 in glioma. Front Pharmacol 9:1503. https:// doi.org/10.3389/fphar.2018.01503

12. Hong Y, Ding Z-Y (2019) PD-1 inhibitors in the advanced esophageal cancer. Front Pharmacol 10:1418. https://doi.org/10.3389/ fphar.2019.01418

13. Shen X, Zhao B (2018) Efficacy of PD-1 or PD-L1 inhibitors and PD-L1 expression status in cancer: meta-analysis. BMJ 362:k3529. https://doi.org/10.1136/bmj.k3529 
14. Wang B-C, Zhang Z-J, Fu C et al (2019) Efficacy and safety of anti-PD-1/PD-L1 agents vs chemotherapy in patients with gastric or gastroesophageal junction cancer: a systematic review and meta-analysis. Medicine (Baltimore) 98(47):e18054. https://doi. org/10.1097/md.0000000000018054

15. Wu X, Gu Z, Chen Y et al (2019) Application of PD-1 blockade in cancer immunotherapy. Comput Struct Biotechnol J 17:661-674. https://doi.org/10.1016/j.csbj.2019.03.006

16. Berghoff AS, Kiesel B, Widhalm G et al (2015) Programmed death ligand 1 expression and tumor-infiltrating lymphocytes in glioblastoma. Neuro Oncol 17(8):1064-1075. https://doi.org/10. 1093/neuonc/nou307

17. Guo L, Zhang H, Chen B (2017) Nivolumab as programmed death-1 (PD-1) inhibitor for targeted immunotherapy in tumor. J Cancer 8(3):410-416. https://doi.org/10.7150/jca.17144

18. ONO receives approval of Opdivo® (Nivolumab) for additional indications of unresectable advanced or recurrent esophageal cancer, and MSI-high unresectable advanced or recurrent colorectal cancer in Japan for a partial change in approved items of manufacturing and marketing approval [press release]. Ono Pharmaceutical Co., Ltd. and Bristol-Myers Squibb Company; Feb 21, 2020. https://www.ono-pharma.com/sites/default/files/en/news/press/ sm_cn200221_1.pdf. Accessed Jan 28, 2021

19. Reardon DA, Brandes AA, Omuro A et al (2020) Effect of nivolumab vs bevacizumab in patients with recurrent glioblastoma: the CheckMate 143 phase 3 randomized clinical trial. JAMA Oncol 6(7):1003-1010. https://doi.org/10.1001/jamaoncol. 2020.1024

20. Adamina M, Tomlinson G, Guller U (2009) Bayesian statistics in oncology. Cancer 115(23):5371-5381. https://doi.org/10.1002/cncr. 24628

21. Berry DA (2012) Bayesian approaches for comparative effectiveness research. Clin Trials (London, England) 9(1):37-47. https:// doi.org/10.1177/1740774511417470

22. Bhattacharjee A (2014) Application of Bayesian approach in cancer clinical trial. World J Oncol 5(3):109-112. https://doi.org/10. 14740/wjon842e

23. Whitehead J, Valdés-Márquez E, Johnson P et al (2008) Bayesian sample size for exploratory clinical trials incorporating historical data. Stat Med 27(13):2307-2327. https://doi.org/10.1002/sim.3140

24. Brookmeyer R, Crowley J (1982) A k-sample median test for censored data. J Am Stat Assoc 77(378):433-440. https://doi.org/10. 1080/01621459.1982.10477829
25. Johnson JM, Bar Ad V, Lorber E, et al (2019) Safety of nivolumab and ipilimumab in combination with radiotherapy in patients with locally advanced squamous cell carcinoma of the head and neck (LA SCCHN). J Clin Oncol 37(15_suppl):6070. https://doi.org/ 10.1200/JCO.2019.37.15_suppl.6070

26. Misch D, de Wit M, Grah C, et al (2017) Efficacy and safety of nivolumab in routine NSCLC treatment - an observational study in 5 lung cancer centers in Berlin, Germany. Eur Resp J 50(suppl 61):OA1477. https://doi.org/10.1183/1393003.congress-2017. OA1477

27. Okada M, Kijima T, Aoe K et al (2019) Clinical efficacy and safety of nivolumab: results of a multicenter, open-label, singlearm, Japanese phase 2 study in malignant pleural mesothelioma (MERIT). Clin Cancer Res 25(18):5485-5492. https://doi.org/10. 1158/1078-0432.ccr-19-0103

28. Omuro A, Vlahovic G, Lim M et al (2018) Nivolumab with or without ipilimumab in patients with recurrent glioblastoma: results from exploratory phase I cohorts of CheckMate 143. Neuro Oncol 20(5):674-686. https://doi.org/10.1093/neuonc/nox208

29. Tamura K, Hasegawa K, Katsumata N et al (2019) Efficacy and safety of nivolumab in Japanese patients with uterine cervical cancer, uterine corpus cancer, or soft tissue sarcoma: multicenter, open-label phase 2 trial. Cancer Sci 110(9):2894-2904. https:// doi.org/10.1111/cas.14148

30. Verzoni E, Cartenì G, Cortesi E et al (2019) Real-world efficacy and safety of nivolumab in previously-treated metastatic renal cell carcinoma, and association between immune-related adverse events and survival: the Italian expanded access program. J Immunother Cancer 7(1):99. https://doi.org/10.1186/ s40425-019-0579-Z

31. Tipping M, Eickhoff J, Robins HI (2017) Clinical outcomes in recurrent glioblastoma with bevacizumab therapy: an analysis of the literature. J Clin Neurosci 44:101-106. https://doi.org/10. 1016/j.jocn.2017.06.070

32. Wong ET, Gautam S, Malchow C et al (2011) Bevacizumab for recurrent glioblastoma multiforme: a meta-analysis. J Natl Compr Canc Netw 9(4):403-407. https://doi.org/10.6004/jnccn.2011.0037

Publisher's Note Springer Nature remains neutral with regard to jurisdictional claims in published maps and institutional affiliations.

\section{Authors and Affiliations}

\section{Tomokazu Aoki $^{1}$ (1) $\cdot$ Naoki Kagawa $^{2} \cdot$ Kazuhiko Sugiyama $^{3} \cdot$ Toshihiko Wakabayashi $^{4} \cdot$ Yoshiki Arakawa $^{5}$. Shigeru Yamaguchi ${ }^{6} \cdot$ Shota Tanaka $^{7} \cdot$ Eiichi Ishikawa $^{8} \cdot$ Yoshihiro Muragaki $^{9} \cdot$ Motoo Nagane $^{10}$. Mitsutoshi Nakada ${ }^{11}$. Satoshi Suehiro ${ }^{12}$. Nobuhiro Hata ${ }^{13}$. Junichiro Kuroda ${ }^{14}$. Yoshitaka Narita ${ }^{15}$. Yukihiko Sonoda ${ }^{16}$. Yasuo Iwadate ${ }^{17}$. Manabu Natsumeda ${ }^{18} \cdot$ Yoichi Nakazato $^{19}$. Hironobu Minami ${ }^{20}$. Yuki Hirata $^{21}$ - Shunsuke Hagihara ${ }^{22} \cdot$ Ryo Nishikawa $^{23}$}

1 Department of Neurosurgery, National Hospital Organization Kyoto Medical Center, 1-1 Fukakusa Mukaihatacho, Fushimi Ward, Kyoto 612-8555, Japan

2 Department of Neurosurgery, Osaka University Graduate School of Medicine, Osaka, Japan

3 Department of Clinical Oncology and Neuro-Oncology Program, Hiroshima University Hospital, Hiroshima, Japan

4 Department of Neurosurgery, Nagoya University Hospital, Nagoya, Japan
5 Department of Neurosurgery, Kyoto University Graduate School of Medicine, Kyoto, Japan

6 Department of Neurosurgery, Hokkaido University Hospital, Hokkaido, Japan

7 Department of Neurosurgery, The University of Tokyo Hospital, Tokyo, Japan

8 Department of Neurosurgery, University of Tsukuba Hospital, Ibaraki, Japan 
9 Department of Neurosurgery, Tokyo Women's Medical University Hospital, Tokyo, Japan

10 Faculty of Medicine, Department of Neurosurgery, Kyorin University, Tokyo, Japan

11 Department of Neurosurgery, Kanazawa University Hospital, Kanazawa, Japan

12 Department of Neurosurgery, Ehime University Hospital, Ehime, Japan

13 Department of Neurosurgery, Graduate School of Medical Sciences, Kyushu University, Fukuoka, Japan

14 Department of Neurosurgery, Kumamoto University Hospital, Kumamoto, Japan

15 Department of Neurosurgery and Neuro-Oncology, National Cancer Center Hospital, Tokyo, Japan

16 Department of Neurosurgery, Yamagata University Hospital, Yamagata, Japan
17 Department of Neurological Surgery, Chiba University Hospital, Chiba, Japan

18 Department of Neurosurgery, Niigata University Medical and Dental Hospital, Niigata, Japan

19 Hidaka Center for Pathologic Diagnosis and Research, Hidaka Hospital, Gunma, Japan

20 Department Medical Oncology/Hematology, Kobe University, Kobe, Japan

21 Oncology Early Clinical Development Planning, Ono Pharmaceutical Co., Ltd, Osaka, Japan

22 Department of Statistical Analysis, Ono Pharmaceutical Co., Ltd, Osaka, Japan

23 Department of Neuro-Oncology/Neurosurgery, Saitama Medical University International Medical Center, Saitama, Japan 